Journal: Environmental Science and Technology Letters

\title{
Adverse Metabolic Outcomes in Soil Pseudomonas Species Exposed to Polyethoxylated Tallow Amine and Glyphosate
}

\author{
Caroll M. Mendonca ${ }^{a}$, Michael L. Reed ${ }^{a}$, Matthew A. Kukurugyaa ${ }^{a}$ Ludmilla Aristildea,b,\#, \\ aDepartment of Biological and Environmental Engineering, College of Agriculture and Life \\ Sciences, Cornell University, Ithaca, NY 14853, USA \\ ${ }^{b}$ Department of Civil and Environmental Engineering, McCormick School of Engineering and \\ Applied Science, Northwestern University, Evanston, Il 60208, USA \\ \#Corresponding Author: ludmilla.aristilde@northwestern.edu (email)
}

\section{SUPPORTING INFORMATION}

Appendix A: Details about culturing Appendix B: Optical Densities at $600 \mathrm{~nm}$ of harvested samples

Appendix C: Protocol for Kinetic Isotopic Switch.

Appendix D: Profiling Extracellular Substrate Depletion

Appendix E: Changes in Biomass Growth Rates

Appendix F: Clustered cellular metabolomes

Appendix G: Metabolite Abbreviations

References 


\section{APPENDIX A}

The method of culturing has been detailed previously. ${ }^{1}$ We grew $25-\mathrm{mL}$ of cell suspensions (up to three biological replicates) in 125-mL baffled flasks placed in a G24 environmental incubator shaker (New Brunswick Scientific, Edison, NJ) operated at $220 \mathrm{rpm}$ and $30^{\circ} \mathrm{C}$. The pH-adjusted ( $\mathrm{pH} \mathrm{7.0)}$ and filter sterilized (0.22 $\mu \mathrm{m}$ nylon; Waters Corporation, Massachusetts) growth medium contained a sole carbon substrate provided as Na-succinate (50 mM) supplemented with major and minor nutrient salts: $89.4 \mathrm{mM} \mathrm{K}_{2} \mathrm{HPO}_{4}, 56.4 \mathrm{mM} \mathrm{NaH}_{2} \mathrm{PO}_{4}, 0.81 \mathrm{mM}$ $\mathrm{MgSO}_{4} \cdot 7 \mathrm{H}_{2} \mathrm{O}, 18.7 \mathrm{mM} \mathrm{NH}_{4} \mathrm{Cl}, 8.6 \mathrm{mM} \mathrm{NaCl}, 34 \mu \mathrm{M} \mathrm{CaCl}_{2} \cdot 2 \mathrm{H}_{2} \mathrm{O}, 30 \mu \mathrm{M} \mathrm{FeSO}_{4} \cdot 7 \mathrm{H}_{2} \mathrm{O}, 0.86 \mu \mathrm{M}$ $\mathrm{CuSO}_{4} \cdot 5 \mathrm{H}_{2} \mathrm{O}, 1.9 \mu \mathrm{M} \mathrm{H}_{3} \mathrm{BO}_{3}, 7.7 \mu \mathrm{MnSO}_{4} \cdot 7 \mathrm{H}_{2} \mathrm{O}, 0.75 \mu \mathrm{M} \mathrm{MnSO}_{4} \cdot 5 \mathrm{H}_{2} \mathrm{O}, 0.26 \mu \mathrm{M} \mathrm{NiCl}_{2} \cdot 6 \mathrm{H}_{2} \mathrm{O}$, and $0.31 \mu \mathrm{M} \mathrm{Na}_{2} \mathrm{MoO}_{4} \cdot 5 \mathrm{H}_{2} \mathrm{O}$. In order to ensure acclimation in each growth condition, For comparisons of data from growth and metabolomics experiments at the three growth conditions, statistical analysis was done using unpaired two-tailed t-test. Statisticallysignificant difference was determined at $p \leq 0.05$. 


\section{APPENDIX B}

Table S1. Optical Densities at $600 \mathrm{~nm}$ of samples harvested during exponential growth of succinate-grown P. putida KT2440, P. putida S12 and P. protegens Pf-5 in the absence (control) and presence POEA $(30 \% \mathrm{w} / \mathrm{w})$ with or without glyphosate $(0.5 \mathrm{mM})$. The measured data were from biological triplicates (trip) $(n=3)$.

\begin{tabular}{|c|c|c|c|c|c|c|c|c|c|}
\hline \multirow[t]{2}{*}{ Condition } & \multicolumn{3}{|c|}{$\begin{array}{l}\text { Pseudomonas putida } \\
\text { KT2440 }\end{array}$} & \multicolumn{3}{|c|}{$\begin{array}{c}\text { Pseudomonas putida } \\
\text { S12 }\end{array}$} & \multicolumn{3}{|c|}{$\begin{array}{c}\text { Pseudomonas protegens } \\
\text { Pf-5 }\end{array}$} \\
\hline & Trip1 & Trip2 & Trip3 & Trip1 & Trip2 & Trip3 & Trip1 & Trip2 & Trip3 \\
\hline Control & 0.96 & 1.03 & 1.01 & 1.05 & 1.02 & 1 & 1.01 & 0.91 & 0.98 \\
\hline POEA & 1.11 & 1.24 & 1.09 & 1.19 & 1.17 & 1.19 & 1.02 & 1.1 & 1.04 \\
\hline POEA + Gly & 1.12 & 1.14 & 1.01 & 1.26 & 1.27 & 1.21 & 1.2 & 1.2 & 1.1 \\
\hline
\end{tabular}




\section{APPENDIX C}

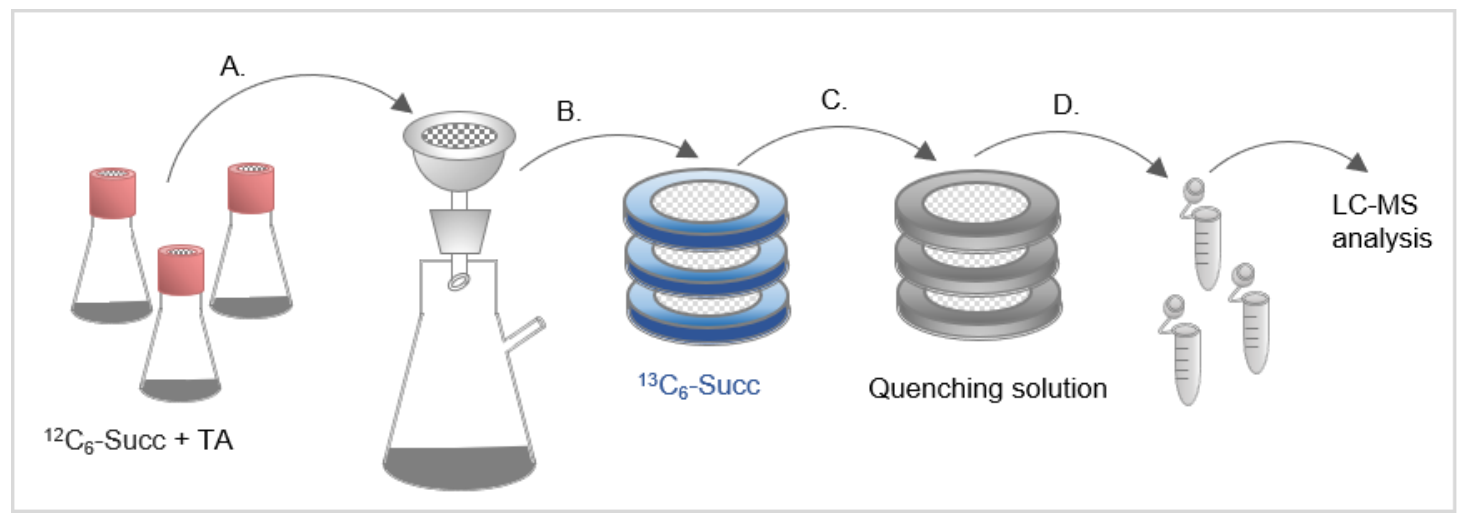

Figure S1. Protocol for kinetic isotopic switch. Batch cultures were grown on a mixture of unlabeled succinate and POEA, and 3-mL of cell suspensions are loaded on filters (A). Filters are transferred to solid agar medium containing ${ }^{13} \mathrm{C}_{6}$-Succinate $(B)$. At specific time points $(0$, 10,30 , and $120 \mathrm{~min}$ ) following the isotopic switch, the metabolites are extracted by submerging cell-containing filters in 2-mL solution of methanol:acetonitrile:water (40:40:20) at $4^{\circ} \mathrm{C}(\mathrm{C})$. Finally, aliquots are prepared as described previously ${ }^{2}$ and in the methods section, prior to LC-MS analysis (D). 


\section{APPENDIX D}

Profiling Extracellular Substrate Depletion. In order to evaluate the effect of POEA (with or without glyphosate) on the extracellular depletion of succinate by the $P$. putida KT2440 cells, we monitored succinate concentration in the growth medium as function of cell growth. Filtered $50-\mu \mathrm{L}$ cell suspensions (three biological replicates) were harvested during lag growth phase (0.05-0.1 $\left.\mathrm{OD}_{600}\right)$, early-exponential growth phase $\left(0.1-1.2 \mathrm{OD}_{600}\right)$, mid-exponential growth phase $\left(1.2-1.8 \mathrm{OD}_{600}\right)$, and stationary phase $\left(>1.8 \mathrm{OD}_{600}\right)$. The succinate concentration was determined by LC-MS analysis. When succinate concentrations were too high for accurate LC-MS analysis, the filtered solutions were diluted with LC-MS grade ultrapure water at 1:10 $\mathrm{v} / \mathrm{v}$ to $1: 1000$ as needed.

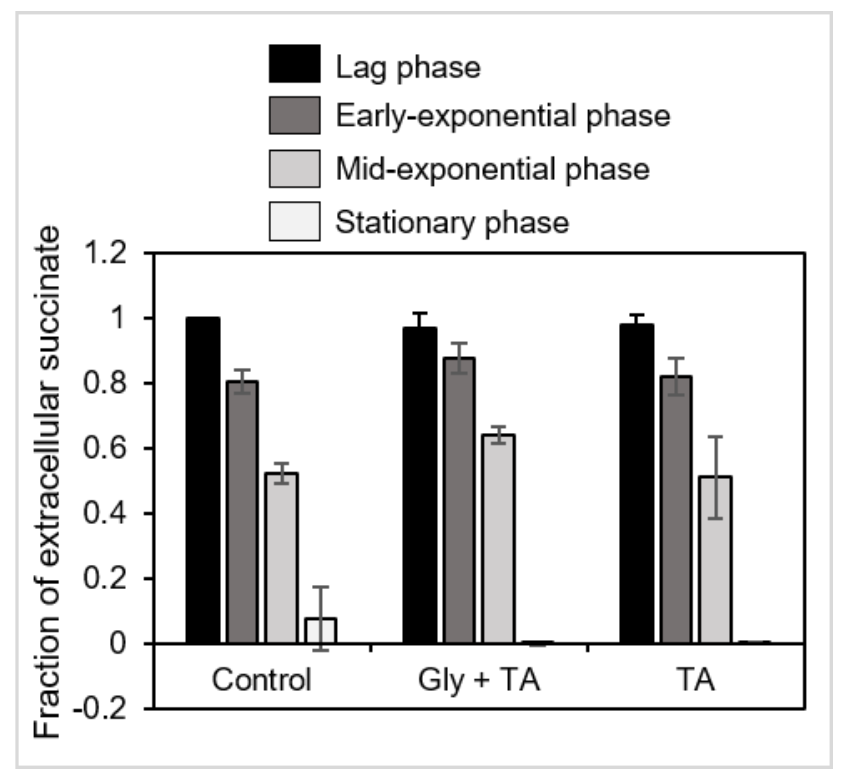

Figure S2. Substrate consumption. Substrate consumption of $P$. putida KT2440 as a function of cell growth during lag, early-exponential, mid-exponential and stationary phase. The measured data were from three biological replicates $(n=3)$. 


\section{APPENDIX E}

Table S2. Percent change in growth rates of succinate-grown $P$. putida KT2440, $P$. putida S12 and P. protegens Pf-5 in the absence (control) and presence POEA (30\% w/w) with or without glyphosate $(0.5 \mathrm{mM})$. The measured data were from three biological replicates $(n=3)$. Twotailed unpaired t-test analysis comparing the specific growth rate of the cells grown on tallow-amine with or without glyphosate.

\begin{tabular}{|cccccc}
\hline & \multicolumn{2}{c}{ POEA } & \multicolumn{2}{c}{ POEA+ Gly } & \\
Bacterial Strain & Average & SD & Average & SD & p-value \\
\hline Pseudomonas putida KT2440 & -58.43 & -1.77 & -63.53 & -2.13 & 0.0017 \\
\hline Pseudomonas putida S12 & -28.48 & -5.60 & -24.48 & -7.33 & 0.5579 \\
\hline Pseudomonas protegens Pf-5 & -0.72 & -7.15 & -16.49 & -3.90 & 0.0032 \\
\hline
\end{tabular}




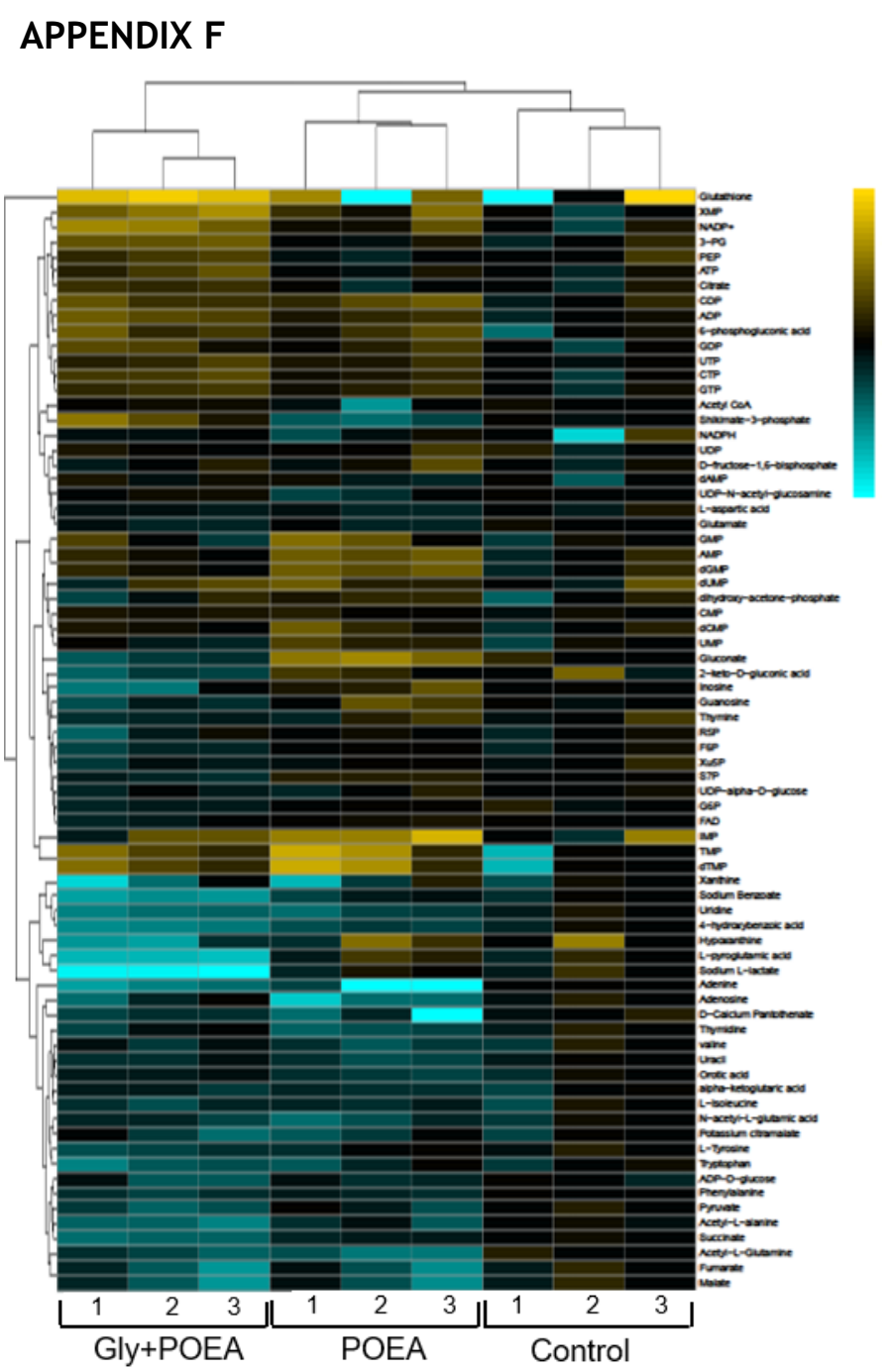

Figure S3. Profiling of the cellular metabolome of $P$. putida KT2440 in the absence of POEA (control) and in the presence of POEA with glyphosate (Gly+POEA) and without glyphosate (POEA). The metabolite levels are presented relative to the median intensities of the control experiments. Measured data from independent biological replicates $(n=3)$ are shown. The data is clustered across both, the metabolites (rows) and the replicates (columns). 


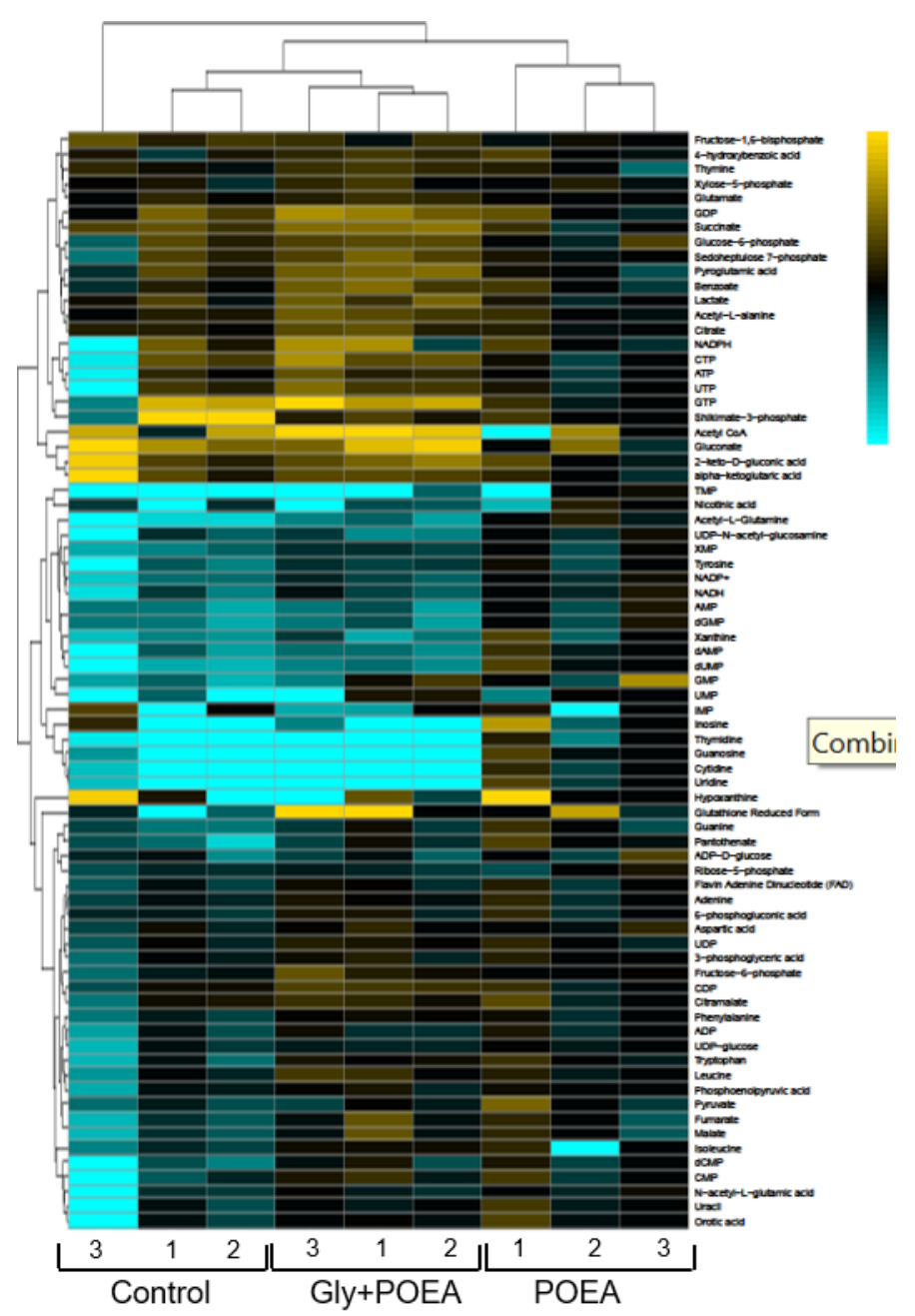

Figure S4. Profiling of the cellular metabolome of $P$. putida S12 in the absence of POEA (control) and in the presence of POEA with glyphosate (Gly+POEA) and without glyphosate (POEA). The metabolite levels are presented relative to the median intensities of the control experiments. Measured data from independent biological replicates $(n=3)$ are shown. The data is clustered across both, the metabolites (rows) and the replicates (columns). 


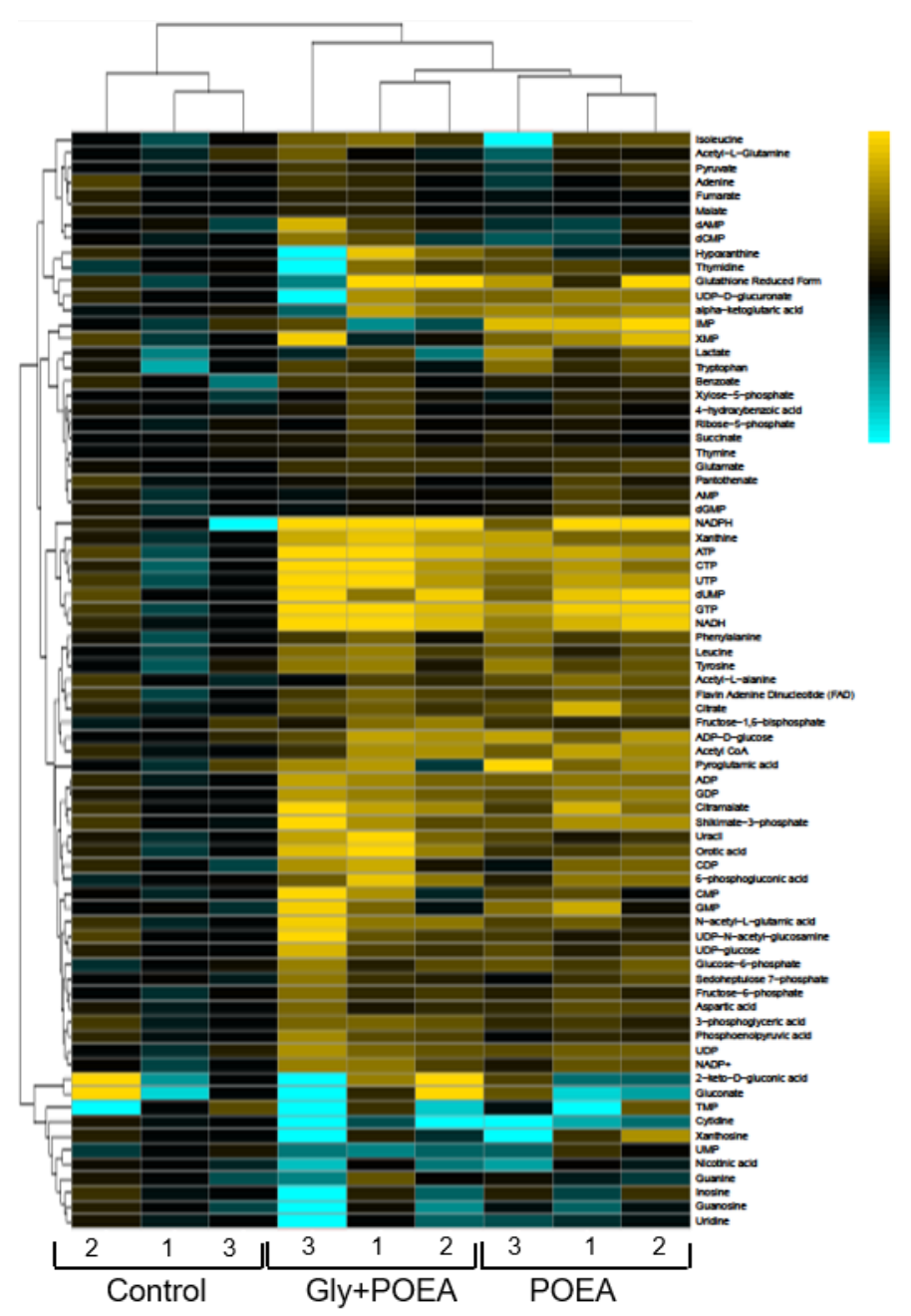

Figure S5. Profiling of the cellular metabolome of $P$. protegens Pf-5 in the absence of POEA (control) and in the presence of POEA with glyphosate (Gly+POEA) and without glyphosate (POEA). The metabolite levels are presented relative to the median intensities of the control experiments. Measured data from independent biological replicates $(n=3)$ are shown. The data is clustered across both, the metabolites (rows) and the replicates (columns). 


\section{Appendix G:}

Table S3. Definition of metabolite abbreviations shown in Figure 2.

\begin{tabular}{|c|c|}
\hline Abbreviation & Description \\
\hline CMP & cytidine monophosphate \\
\hline GMP & guanosine monophosphate \\
\hline AMP & adenosine monophosphate \\
\hline dGMP & deoxy-guanosine monophosphate \\
\hline dCMP & deoxy-cytidine monophosphate \\
\hline UMP & uridine monophosphate \\
\hline ADP & adenosine diphosphate \\
\hline CDP & cytidine diphosphate \\
\hline 6-PG & 6-phosphogluconate \\
\hline IMP & inosine monophosphate \\
\hline dUMP & deoxy-uridine monophosphate \\
\hline ATP & adenosine triphosphate \\
\hline dAMP & deoxy-adenosine monophosphate \\
\hline GTP & guanosine triphosphate \\
\hline UTP & uridine triphosphate \\
\hline CTP & cytidine triphosphate \\
\hline XMP & xanthosine monophosphate \\
\hline GDP & guanosine diphosphate \\
\hline $\mathrm{NADP}+$ & nicotinamide adenine dinucleotide phosphate \\
\hline UDP & uridine diphosphate \\
\hline FBP & fructose 1,6-bisphosphate \\
\hline NADPH & nicotinamide adenine dinucleotide phosphate [reduced] \\
\hline 3-PG & 3-phosphoglycerate \\
\hline PEP & phosphoenolpyruvate \\
\hline G6P & glucose 6-phosphate \\
\hline F6P & fructose 6-phosphate \\
\hline S7P & sedoheptulose 7-phosphate \\
\hline Xu5P & xylulose 5-phosphate \\
\hline $\mathrm{R} 5 \mathrm{P}$ & ribose 5-phosphate \\
\hline FAD & flavin adenine dinucleotide \\
\hline
\end{tabular}




\section{References}

(1) Aristilde, L.; Reed, M. L.; Wilkes, R. A.; Youngster, T.; Kukurugya, M. A.; Katz, V.; Sasaki, C. R. S. Glyphosate-Induced Specific and Widespread Perturbations in the Metabolome of Soil Pseudomonas Species. Front. Environ. Sci. 2017, 5 (June), 1-13. https://doi.org/10.3389/fenvs.2017.00034.

(2) Yuan, J.; Bennett, B. D.; Rabinowitz, J. D. Kinetic Flux Profiling for Quantitation of Cellular Metabolic Fluxes. Nat. Protoc. 2008, 3 (8), 1328-1340. https://doi.org/10.1038/nprot.2008.131. 